\title{
Representatividad de los tipos de vegetación en distintas iniciativas de conservación en Oaxaca, México
}

\author{
Representativeness of vegetation types in different conservation initiatives in Oaxaca, Mexico
}

\author{
Alina Gabriela Monroy Gamboa a*, Víctor Sánchez-Cordero a , Miguel Briones-Salas ${ }^{\text {b, }}$ \\ Rafael Lira-Saade c, José Manuel Maass Moreno ${ }^{\text {d }}$ \\ *Autor de correspondencia: a Universidad Nacional Autónoma de México, Instituto de Biología, Departamento de Zoología, \\ Laboratorio de Sistemas de Información Geográfica, Circuito Exterior s/n, Ciudad Universitaria, Coyoacán, Distrito Federal, \\ México, C.P. 04510, tel.: (0052-55) 5622-9147, ext. 47846, beu_ribetzin@hotmail.com \\ b Instituto Politécnico Nacional, Centro Interdisciplinario de Investigación para el Desarrollo Integral Regional-Oaxaca, \\ Laboratorio de Vertebrados Terrestres (Mastozoología), Oaxaca, México. \\ c Universidad Nacional Autónoma de México, Facultad de Estudios Superiores-Iztacala, \\ Laboratorio de Recursos Naturales, UBIPRO, Estado de México, México. \\ ${ }^{d}$ Universidad Nacional Autónoma de México, Centro de Investigaciones en Ecosistemas-Morelia, Michoacán, México.
}

\begin{abstract}
SUMMARY
Oaxaca shows an exceptional biodiversity, although a high rate of natural habitat loss threatens its conservation. Here we analyze different initiatives for conservation, their extension and vegetation representativeness in Oaxaca, until 2008. We compiled different initiatives from governmental, non-governmental, and academic and private institutions and analyzed their geographic overlap. Conservation initiatives were classified in three groups: two initiatives with support and recognition of the Mexican government (official conservation initiatives) and a third one stemming from indigenous communities known as voluntarily preserved areas. The results showed that the official conservation initiatives covered approximately $40 \%$ of Oaxaca, showing $19 \%$ of overlap. Official conservation initiatives adequately represented pine-oak forests; nonetheless, open forests, with secondary vegetation of shrubs and herbs are poorly represented. The conservation initiative showing the highest extension were communities supported by the Payment for Environmental Services Program, while the voluntarily preserved areas had the smallest extension in Oaxaca.
\end{abstract}

Key words: voluntarily preserved areas, initiatives of conservation, Mexico, vegetation types, biodiversity.

\section{RESUMEN}

El estado de Oaxaca al sur de México, posee una excepcional biodiversidad, pero una alta pérdida de hábitat natural amenaza su conservación. Este estudio presenta un análisis de las distintas iniciativas de conservación existentes, su extensión territorial y los tipos de vegetación que representan en Oaxaca, con base en consultas a diversas organizaciones gubernamentales y no gubernamentales, instituciones académicas y privadas, así como en literatura científica publicada hasta el año 2008. Se analizó, por medio de un sistema de información geográfica, la superposición de las diversas iniciativas de conservación. Estas iniciativas se clasificaron en tres grupos: dos iniciativas con respaldo y reconocimiento de parte del gobierno mexicano y una más constituida por iniciativas particulares de comunidades indígenas, denominadas áreas voluntarias para la conservación. Los resultados indicaron que los tres tipos de iniciativas de conservación, en conjunto, ocupan aproximadamente $40 \%$ de la superficie de Oaxaca; se observó una sobreposición de $19 \%$ de la superficie entre estas iniciativas, principalmente en tierras cubiertas por bosque de pino-encino. No se detectaron iniciativas de conservación en el bosque bajo-abierto con vegetación secundaria arbustiva y herbácea. La iniciativa que abarcó la mayor extensión territorial en Oaxaca fue la de las comunidades apoyadas por el Programa de Pago por Servicios Ambientales, en tanto que las áreas voluntarias de conservación, se caracterizan por haber cubierto la menor extensión territorial de Oaxaca.

Palabras clave: áreas voluntarias de conservación, iniciativas de conservación, México, tipos de vegetación, biodiversidad.

\section{INTRODUCCIÓN}

La enorme pérdida de biodiversidad a nivel mundial, incrementa el interés por la generación de diversas iniciativas de conservación, principalmente en países megadiversos (Mittermeier et al. 1997). Entre estos países se encuen- tra México que, desde hace varios años, ha decretado una serie de áreas naturales protegidas en gran parte de su territorio (CONANP 2008). La riqueza biológica de México se concentra, principalmente, en su parte correspondiente a la región Neotropical, donde se ubica Oaxaca, uno de los estados con mayor diversidad biológica del país. Oaxaca in- 
cluye el $50 \%$ de plantas vasculares, $19 \%$ de invertebrados, $35 \%$ de anfibios, $26 \%$ de reptiles, $63 \%$ de aves y $55 \%$ de los mamíferos terrestres de México, así como 127 especies de peces continentales; adicionalmente, estas especies tienen una importancia socioeconómica relevante, ya que más de 500 especies de plantas y 85 especies de insectos comestibles son usados frecuentemente en Oaxaca (García-Mendoza et al. 2004). Se reconocen 26 diferentes tipos de vegetación agrupados bajo un criterio fisonómico-florístico (García-Mendoza et al. 2004). Sus diversos tipos de bosques cubren cerca del $64 \%$ del estado $(9,5$ millones de hectáreas) y cerca del $70 \%$ de este territorio corresponde a comunidades forestales, de las cuales 3,3 millones de hectáreas corresponden a bosques templados y 2,6 millones de hectáreas a bosques húmedos o bosques secos. El $82 \%$ de ese territorio es propiedad de comunidades indígenas o mestizas (Martin et al. 2011). Oaxaca muestra un acelerado cambio de uso de suelo que ha resultado en una alta pérdida de la cobertura vegetal debido al pastoreo excesivo, la expansión agrícola, el crecimiento de los asentamientos humanos y la explotación irracional de los bosques, con la consecuente amenaza de pérdida de la biodiversidad. El deterioro de los ecosistemas puede resultar en extinción de especies y en la pérdida del uso sustentable de los recursos bióticos (García-Mendoza et al. 2004).

Las iniciativas de conservación de la biodiversidad que el gobierno federal ha establecido en el país y, consecuentemente, en Oaxaca son (1) áreas protegidas decretadas, consideradas como el instrumento de política de conservación de biodiversidad más importante en México, reguladas por la Comisión Nacional de Áreas Naturales Protegidas, y que son porciones terrestres o acuáticas que representen ecosistemas relevantes, donde el ambiente original no ha sido esencialmente alterado o que produzca algún beneficio ecológico (LGEEPA 2012). Actualmente, existen 174 áreas naturales protegidas en el país, de las cuales, siete se encuentran en Oaxaca (CONANP 2007); (2) áreas protegidas, bajo el esquema de pagos por servicios ambientales, principalmente pago por servicios hidrológicos, captura de carbono y biodiversidad, administrados por la Comisión Nacional Forestal; bajo este esquema, las comunidades indígenas y campesinas, y pequeños propietarios son apoyados económicamente por ofrecer cuidados a sus bosques; estos pueden clasificarse en protección de la biodiversidad (no extracción ni cacería) e hidrológicos, destinados mantener la cobertura vegetal y manejar adecuadamente los suelos para contribuir a la conservación y recarga de los acuíferos y (3) Unidades de Manejo para la Conservación de Vida Silvestre, que buscan promover esquemas alternativos de producción compatibles con el cuidado del ambiente, a través del uso racional, ordenado y planificado de los recursos naturales renovables contenidos en ellas, frenando o revirtiendo los procesos de deterioro ambiental (LGEEPA 2012).

Además de estos esfuerzos federales de conservación, existen otras dos iniciativas importantes a nivel estatal:
(1) áreas protegidas estatales decretadas, que incluyen áreas con uno o más ecosistemas, que poseen belleza escénica, valor científico, educativo, recreacional, histórico y con flora y fauna, con aptitud para el desarrollo del turismo (CONANP 2007). Son decretadas y administradas enteramente por el gobierno estatal y hasta el momento, se pueden reconocer como ejemplo de este esquema de conservación: un monumento natural, dos santuarios y un área de protección de flora y fauna (Martin et al. 2011), y (2) áreas voluntarias de conservación, las cuales pertenecen a comunidades indígenas, campesinos, particulares y empresas que de manera voluntaria son destinadas a la conservación. Estas últimas están, a su vez, divididas en áreas de conservación certificadas, las cuales son reconocidas por la CONANP y se integran al sistema de áreas naturales de conservación en Oaxaca, sin que las comunidades pierdan el dominio, manejo o gobernanza de las tierras que destinan a conservación (Elizondo y López 2009) y, finalmente, áreas comunales protegidas, que son áreas comunitarias indígenas y campesinas, sin un registro o certificación oficial; en la mayoría de los casos estas áreas son seleccionadas de acuerdo con un sistema muy antiguo de "usos y costumbres", a través de asambleas comunitarias.

Es importante mencionar que existen aproximadamente 150 áreas comunales protegidas en México, la mayoría de las cuales se ubican en Oaxaca (CONABIO 2008, Martin et al. 2011). Lo anterior resulta lógico, debido a que $80 \%$ de la superficie forestal de Oaxaca, así como la biodiversidad que sus ecosistemas albergan se encuentran bajo el control de aproximadamente 1.400 comunidades indígenas ${ }^{1}$ y ejidos ${ }^{2}$ (Moguel y Toledo 1999, Sarukhán y Larson 2001, Martin et al. 2011, DOF 1992).

Este grupo de áreas comunales de conservación, están definidas por la International Union for Conservation of Nature como "ecosistemas naturales y/o modificados incluyendo valores de biodiversidad, beneficios ecológicos y valores culturales conservados voluntariamente por indígenas y comunidades locales, sedentarias y móviles, a través de leyes consuetudinarias y otros significados efectivos" (Borrini-Feyerabend et al. 2004). Dentro de esta categoría se pueden distinguir las áreas de conservación por manejo forestal, las reservas comunales campesinas, los sitios naturales sagrados, las áreas de conservación bajo cafetales, las áreas destinadas al ecoturismo, las áreas destinadas a

Comunidades indígenas: núcleos de poblaciones formados por el conjunto de tierras, bosques y aguas que fueron reconocidos o restituidos, y que han estado en posesión de esta población desde muchos años atrás, con costumbres y prácticas comunales.

Ejidos: parcelas de tierra, bosques o aguas que el gobierno reconoce como personalidad jurídica y protege su propiedad sobre la tierra, tanto para el asentamiento humano y regulará el aprovechamiento de tierras, bosques y aguas de uso común y la provisión de acciones de fomento necesarias para elevar el nivel de vida de sus pobladores. Los derechos parcelarios se pueden transmitir entre los miembros del núcleo de población y el Estado fijará los requisitos y procedimientos conforme a los cuales la asamblea ejidal otorgará al ejidatario el dominio sobre su parcela. 
proyectos productivos y las áreas para servicios ambientales locales, entre otras (Borrini-Feyerabend et al. 2004).

La Comisión Nacional de Áreas Naturales Protegidas (CONANP) ha promovido la certificación de aproximadamente 177 áreas desde 2002, ya sean comunitarias o privadas a solicitud de los dueños, en el marco normativo de la Ley General del Equilibrio Ecológico y Protección al Ambiente, que establece que las comunidades indígenas y campesinas pueden establecer áreas de conservación de manera legal y voluntaria. En Oaxaca, existen 74 áreas de conservación certificadas, comunales o ejidales y 36 privadas (CONANP 2007, Ortega-del Valle et al. 2010).

Estas iniciativas de conservación, en conjunto, intentan conocer, proteger y utilizar la biodiversidad de uno de los estados más ricos biológicamente de México. Sin embargo, no se sabe con exactitud su extensión y funcionalidad. En este sentido, se plantea como interrogante, si la iniciativa de conservación propuesta por el Gobierno Federal (Áreas Protegidas decretadas) es suficiente para representar, adecuadamente la diversidad de tipos de vegetación presentes en el estado de Oaxaca. El planteamiento alternativo es que es indispensable incluir a todas las diferentes iniciativas de conservación para representar adecuadamente la diversidad de tipos de vegetación de Oaxaca.

Los objetivos de este trabajo son (1) presentar un análisis cualitativo de la distribución geográfica, extensión territorial y representatividad de los tipos de vegetación de las distintas iniciativas de conservación en Oaxaca, (2) analizar la distribución geográfica de las iniciativas de conservación (áreas naturales protegidas, áreas apoyadas con pagos por servicios ambientales y áreas voluntarias de conservación) en las provincias fisiográficas del estado y los tipos de vegetación presentes en las zonas de superposición y de ausencia de iniciativas de conservación y, (3) analizar la contribución de las áreas voluntarias de conservación respecto a la representación de los tipos de vegetación de Oaxaca.

\section{MÉTODOS}

Oaxaca es el quinto estado más extenso en México, con una superficie de $95.364 \mathrm{~km}^{2}$, que representa 4,8\% de la superficie del país. Sus coordenadas geográficas extremas son: $15^{\circ} 39^{\prime}-18^{\circ} 42^{\prime} \mathrm{N}$ y $93^{\circ} 52^{\prime}-98^{\circ} 32^{\prime} \mathrm{O}$. La variación altitudinal oscila desde el nivel del mar hasta los $3.750 \mathrm{~m}$ s.n.m. y se caracteriza por su fisiografía accidentada, diversos tipos de clima y de suelos, que propicia una alta diversidad biológica y de comunidades vegetales. Se reconocen en el estado 26 diferentes tipos de vegetación, agrupados bajo un criterio fisonómico-florístico. La paleografía demuestra que el estado fue desplazado desde una latitud mayor a la actual, lo que junto con el intricado relieve, la exposición a los sistemas meteorológicos que se desarrollan en el Golfo de México y en el Océano Pacífico, las corrientes marinas y la temperatura del océano, hacen que Oaxaca se distinga por tener una alta heterogeniedad ambiental y variedad climática. Oaxaca también tiene una compleja historia edafológica; las condiciones de los suelos, los hacen vulnerables a la erosión natural, por sus ríos y microcuencas con pendientes pronunciadas (GarcíaMendoza et al. 2004).

Durante el año 2008, se realizaron consultas a quince instituciones involucradas en alguna acción de conservación dentro del estado, para obtener información biológica, estadística y geográfica de las áreas que cuentan con alguna de estas iniciativas. Dicha consulta, se hizo mediante entrevistas en las que los informantes hablaron libremente sobre el tema de las iniciativas de conservación en el estado y proporcionaron documentos que fueron revisados, como ordenamientos territoriales, mapas y polígonos de áreas de conservación. Del Gobierno Federal se consultaron documentos de ocho instituciones del sector ambiental: La Secretaría del Medio Ambiente y Recursos Naturales, el Instituto Nacional de Ecología, la Procuraduría Federal de Protección al Ambiente, la Comisión Nacional para el Conocimiento y Uso de la Biodiversidad, la Comisión Nacional de Áreas Naturales Protegidas, la Comisión Nacional Forestal, la Comisión Nacional del Agua y el Instituto Mexicano de Tecnología del Agua. De estas, solamente se utilizaron los datos de cuatro que cumplían con los objetivos del estudio y fueron más completos y confiables: la Secretaría del Medio Ambiente y Recursos Naturales, la Comisión Nacional para el Conocimiento y Uso de la Biodiversidad, la Comisión Nacional de Áreas Naturales Protegidas y la Comisión Nacional Forestal.

En cuanto a instituciones no gubernamentales se realizaron consultas solamente en cuatro de ellas, las cuales contaban con información disponible: dos internacionales (World Wildlife Fund y Global Diversity Fundatio) y dos nacionales (Geoconservación Asociación Civil y Estudios Rurales y Asesoría Campesina, Asociación Civil). De las instituciones educativas y centros de investigación, se visitaron las tres más importantes en cuanto a antigüedad y producción científica sobre el tema de las iniciativas de conservación en el estado de Oaxaca: el Centro Interdisciplinario de Investigación para el Desarrollo Integral Regional (Unidad Oaxaca, Instituto Politécnico Nacional), el Instituto Tecnológico del Valle de Oaxaca y a la Universidad Benito Juárez de Oaxaca; sin embargo, en estas últimas dos, la información fue escasa.

Los datos geográficos proporcionados por las diferentes instituciones, como los polígonos de las iniciativas de conservación, se analizaron por medio de un sistema de información geográfica, mediante el programa ArcView (3.2). En primer lugar, se proyectaron sobre el polígono del estado de Oaxaca. En el caso de áreas protegidas que además abarcan a otros estados, como la Reserva de la Biosfera Tehuacán-Cuicatlán, los polígonos se cortaron siguiendo el contorno del límite estatal para obtener la superficie que ocupan dentro de Oaxaca.

Todas las iniciativas de conservación identificadas se clasificaron en tres grandes grupos, considerando prin- 
cipalmente el tipo de administración: a) Áreas naturales protegidas: se incluyen aquellas áreas que cuentan con un decreto y son de administración estatal o federal; b) Áreas apoyadas con pagos por servicios ambientales, por el gobierno federal, durante los años 2003-2007 y por biodiversidad e hidrológicos durante 2008: agrupa a todas aquellas áreas que fueron propuestas por los dueños de los terrenos a cambio de un incentivo económico por parte del gobierno federal; y c) Áreas voluntarias para la conservación: incluye todas las áreas donde las comunidades indígenas o campesinas, así como particulares o empresas forestales decidieron proteger alguna parte de su territorio de manera voluntaria. Se incluyen aquellas áreas que han sido certificadas por la Comisión Nacional de Áreas Naturales Protegidas (comunales, ejidales y privadas o particulares) así como las que no cuentan con certificación. En ambos casos el cuidado, administración y uso están exclusivamente a cargo de los dueños de las áreas.

Para cada área identificada de manera individual se obtuvieron las coordenadas geográficas $y$, a partir de ellas se generó su respectivo polígono (en proyección cónica conforme de Lambert). Se cuantificó el número de áreas para cada categoría y se obtuvo la extensión territorial para el conjunto de ellas. Se cuantificó la superficie $\left(\mathrm{km}^{2}\right)$ superpuesta de los polígonos entre las diversas iniciativas y el porcentaje con respecto al territorio estatal. Así mismo, se proyectaron estas áreas sobre el mapa de uso de suelo y vegetación (serie III del Instituto Nacional de Estadística y Geografía; INEGI 2007) con lo que se obtuvo el porcentaje de superficie que correspondiente a cada tipo de vegetación, representados en las iniciativas de conservación. De las 45 categorías de vegetación que se encontraron representadas dentro del estado de Oaxaca, se eliminaron nueve (agricultura de humedad, agricultura de riego y riego eventual, agricultura de temporal con cultivos anuales, agricultura de temporal con cultivos permanentes y semipermanentes, áreas sin vegetación aparente, asentamientos humanos, pastizal cultivado, pastizal inducido y cuerpos de agua) debido a que estas categorías no representan tipos de vegetación.

Por otra parte, se analizó la ubicación geográfica de las iniciativas de conservación en cada una de las 12 provincias fisiográficas del estado (figura 1; Ortíz-Pérez et al. 2004) y se cuantificó el número de ellas presentes en cada provincia, así como la extensión territorial que cuenta (o no) con iniciativas de conservación. Se analizó si existen diferencias significativas entre las extensiones que cubren los tipos de vegetación en las diferentes iniciativas de conservación, por medio de un análisis de Varianza de Medidas Repetidas. Posteriormente, para separar los grupos que difieren

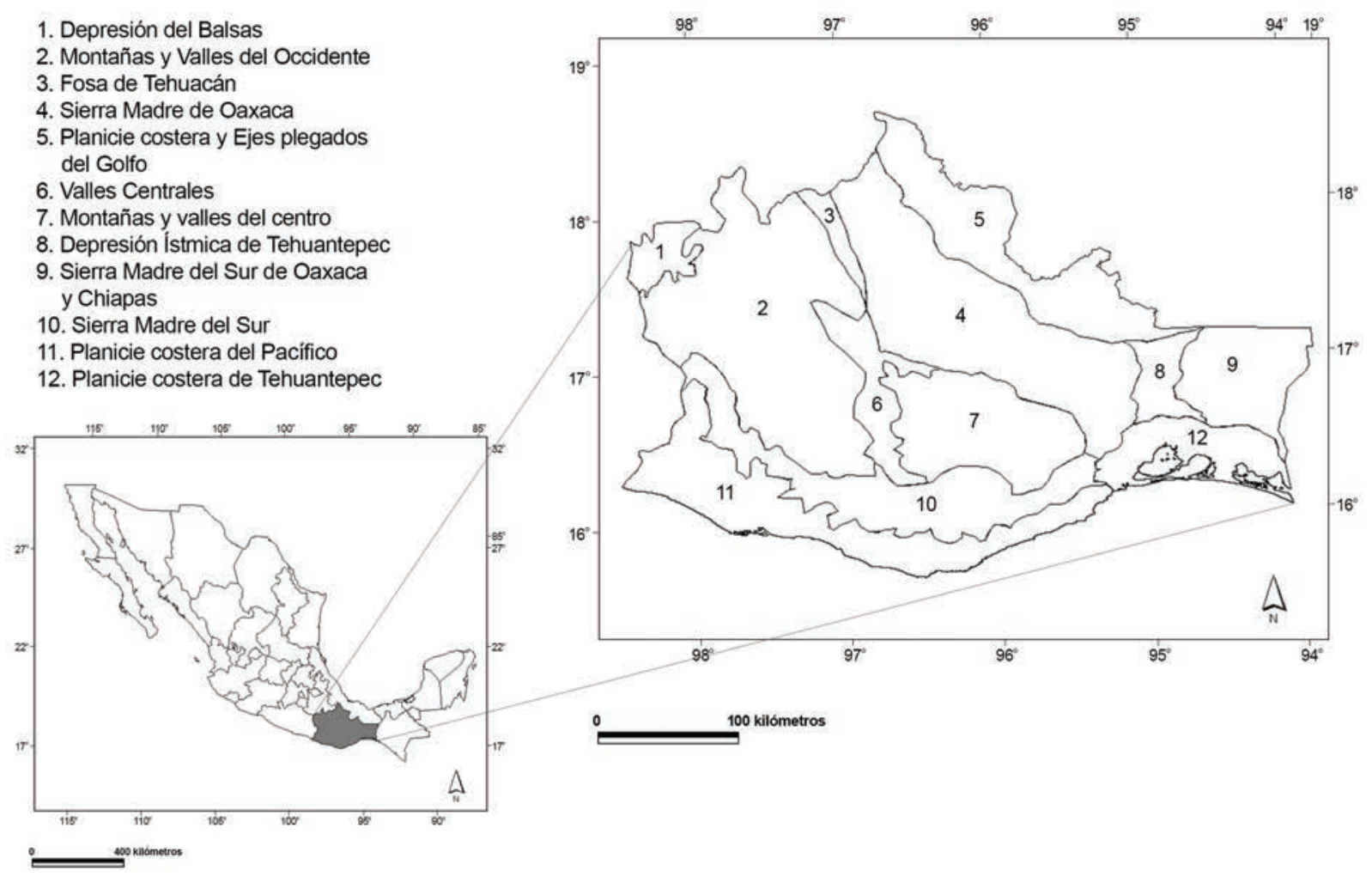

Figura 1. Ubicación geográfica de Oaxaca, México, y la división del estado en provincias fisiográficas. Modificado de Ortíz-Pérez et al. (2004).

Geographic localization of Oaxaca in Mexico and the state division by physiographic provinces. Modified from Ortíz-Pérez et al. (2004). 
de otros, se realizó una comparación múltiple, utilizando la prueba de Student-Newman, con un nivel de probabilidad de $P<0,05$, en el programa SigmaStat 3.5 (Zar 1984).

\section{RESULTADOS}

Se registró un total de 880 áreas bajo alguna iniciativa de conservación. La mayoría (337) de estas pertenecen a la categoría de comunidades apoyadas con pagos por servicios ambientales hidrológicos. Las comunidades apoyadas con pagos por servicios ambientales, cubrieron poco más de la mitad del territorio del estado (52,32\%). Por su parte, las áreas naturales protegidas decretadas por el gobierno federal, cubrieron $3.256,07 \mathrm{~km}^{2}(3,41 \%$ con respecto al estado). La extensión territorial de las áreas voluntarias de conservación sumó una superficie similar a la de las áreas naturales protegidas. Las áreas comunales protegidas sin certificación contribuyeron con $1.073,7 \mathrm{~km}^{2}(1,1 \%$ de Oaxaca), las áreas comunales o ejidales certificadas, un total de $921,3 \mathrm{~km}^{2}$ (0,9\% de Oaxaca); en tanto que las áreas naturales protegidas decretadas por el gobierno estatal tuvieron una superficie de $41,75 \mathrm{~km}^{2}(0,04 \%$ de la superficie del estado) y finalmente las áreas certificadas privadas 9,9 $\mathrm{km}^{2}(0,01 \%$ de Oaxaca). El total de las tres iniciativas de conservación incluyeron un área de $54.621,1 \mathrm{~km}^{2}$ que equivale poco más de la mitad del territorio Oaxaqueño (cuadro 1, figura 2).
La provincia conocida como Depresión del Balsas contó sólo con una iniciativa de conservación de pago por servicios ambientales hidrológicos, seguida de la planicie costera de Tehuantepec con dos iniciativas, la cual se considera una zona de gran importancia de distribución de especies vegetales y de fauna. En contraste, se encontró que la provincia de la Sierra Madre de Oaxaca es en la que

Cuadro 1. Superficie cubierta por las iniciativas de conservación en Oaxaca, México y su porcentaje con respecto a la superficie total del estado.

Area covered by conservation initiatives in Oaxaca, Mexico, and their percentage regarding the total state's area.

\begin{tabular}{lcc}
\hline $\begin{array}{l}\text { Iniciativa de } \\
\text { conservación }\end{array}$ & $\begin{array}{c}\text { Superficie } \\
\left(\mathrm{km}^{2}\right)\end{array}$ & Porcentaje \\
\hline $\begin{array}{l}\text { Áreas apoyadas con } \\
\text { pagos por servicios } \\
\text { ambientales }\end{array}$ & $50.761,9$ & 53,2 \\
$\begin{array}{l}\text { Áreas naturales } \\
\text { protegidas }\end{array}$ & $3.927,8$ & 3,5 \\
$\begin{array}{l}\text { Áreas voluntarias de } \\
\text { conservación }\end{array}$ & $2.004,9$ & 2,1 \\
\hline Total & $54.621,1$ & 57,3 \\
\hline
\end{tabular}

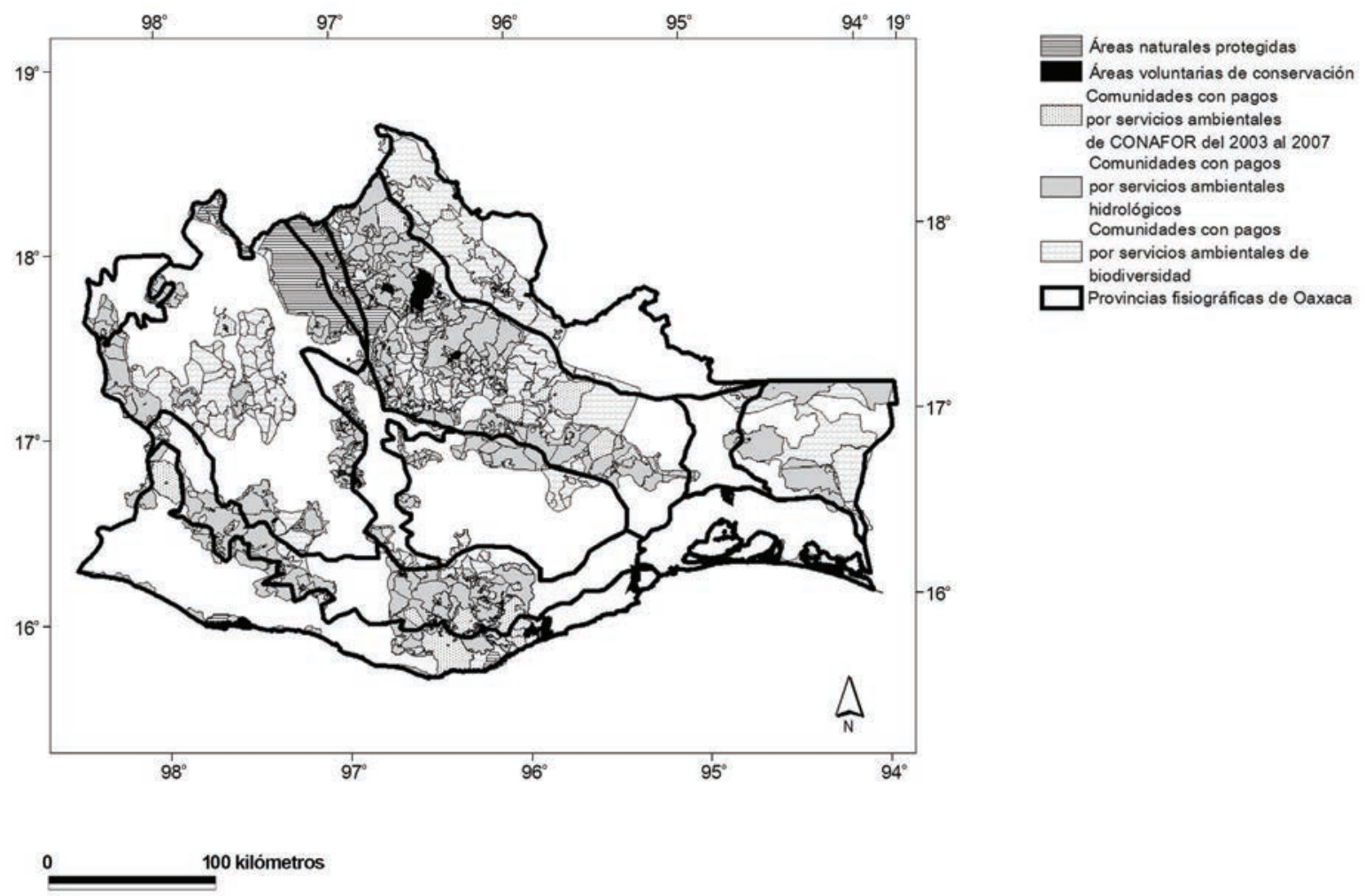

Figura 2. Localización de las iniciativas de conservación en el estado de Oaxaca, México.

Localization of the conservation initiatives of Oaxaca, Mexico. 
se conserva una superficie mayor y en ella estaban representadas todas las diferentes iniciativas de conservación (cuadro 2).

Las comunidades que recibieron pago por servicios ambientales hidrológicos, mostraron la mayor superposición con todas las demás iniciativas, seguidas por las comunidades que reciben pagos por servicios ambientales de biodiversidad. Las únicas iniciativas de conservación que no se sobrepusieron, fueron las áreas protegidas federales con las áreas voluntarias de conservación, debido a que estas últimas son de iniciativa y financiamiento comunal o ejidal (cuadro 3). La superposición de iniciativas de conservación mostró que los esfuerzos se multiplicaron en algunas áreas, como ocurrió en la Sierra Madre de Oaxaca y Sierra Madre del Sur, mientras que otras se encontraron relativamente desprotegidas, propiciando que algunas especies endémicas no contaran con este tipo de protección.
Las iniciativas de conservación, en su conjunto, incluyeron 35 tipos de vegetación de los 36 considerados en el mapa de la serie III de vegetación y uso de suelo para el estado de Oaxaca (INEGI 2007). El bosque de pino-encino y encino-pino, fue el tipo de vegetación mejor representado en las diversas iniciativas de conservación $(73,8 \%)$, mientras que los de menor representación $(0,02 \%)$ fueron el popal-tular (vegetación primaria) y la selva baja espinosa con vegetación secundaria arbustiva y herbácea (vegetación secundaria). El único tipo de vegetación que no estuvo representado en ninguna de las iniciativas de conservación fue el bosque abierto con vegetación secundaria arbustiva y herbácea (cuadro 4).

Los tipos de vegetación que cuentan con una extensión menor al $0,05 \%$ en las diversas iniciativas de conservación son el bosque bajo-abierto con vegetación secundaria arbustiva y herbácea, selva baja espinosa con vegetación

Cuadro 2. Superficie $\left(\mathrm{km}^{2}\right)$ ocupada por las diferentes iniciativas de conservación para cada provincia fisiográfica y el número de las mismas (en paréntesis) en el estado de Oaxaca, México.

Surface $\left(\mathrm{km}^{2}\right)$ of the different conservation initiatives for each physiographic province and the number of them (between brackets) at Oaxaca State, Mexico.

\begin{tabular}{|c|c|c|c|c|c|c|}
\hline $\begin{array}{l}\text { Provincia } \\
\text { fisiográfica }\end{array}$ & $\begin{array}{l}\text { Superficie } \\
\text { total }\end{array}$ & $\begin{array}{c}\text { Áreas } \\
\text { naturales } \\
\text { protegidas }\end{array}$ & $\begin{array}{c}\text { Comunidades } \\
\text { apoyadas con } \\
\text { pagos por servicios } \\
\text { ambientales } \\
(2003-2007)\end{array}$ & $\begin{array}{l}\text { Comunidades } \\
\text { apoyadas con } \\
\text { pagos por servicios } \\
\text { ambientales } \\
\text { (Hidrológicos) }\end{array}$ & $\begin{array}{c}\text { Comunidades } \\
\text { apoyadas con } \\
\text { pagos por servicios } \\
\text { ambientales } \\
\text { (Biodiversidad) }\end{array}$ & $\begin{array}{c}\text { Áreas } \\
\text { voluntarias de } \\
\text { conservación }\end{array}$ \\
\hline $\begin{array}{l}\text { Depresión del } \\
\text { Balsas }\end{array}$ & 1.660 & 0 & 0 & $\begin{array}{l}412 \\
(18)\end{array}$ & 0 & 0 \\
\hline $\begin{array}{l}\text { Montañas y Valles } \\
\text { del Occidente }\end{array}$ & 19.993 & $\begin{array}{r}1.695 \\
(1)\end{array}$ & $\begin{array}{l}348 \\
(25)\end{array}$ & $\begin{array}{r}3.237 \\
(80)\end{array}$ & $\begin{array}{r}5.416 \\
(25)\end{array}$ & 0 \\
\hline Fosa de Tehuacán & 1.068 & $\begin{array}{r}865 \\
(1)\end{array}$ & $\begin{array}{l}85 \\
(7)\end{array}$ & $\begin{array}{l}83 \\
(8)\end{array}$ & $\begin{array}{l}917 \\
(16)\end{array}$ & 0 \\
\hline $\begin{array}{l}\text { Sierra Madre de } \\
\text { Oaxaca }\end{array}$ & 17.098 & $\begin{array}{r}427 \\
(1)\end{array}$ & $\begin{array}{l}2.696 \\
(115)\end{array}$ & $\begin{array}{l}8.759 \\
(136)\end{array}$ & $\begin{array}{r}5.397 \\
(25)\end{array}$ & $\begin{array}{r}282 \\
(6)\end{array}$ \\
\hline $\begin{array}{l}\text { Planicie Costera y } \\
\text { Ejes plegados del } \\
\text { Golfo }\end{array}$ & 9.713 & 0 & $\begin{array}{r}193 \\
(9)\end{array}$ & $\begin{array}{l}190 \\
(10)\end{array}$ & $\begin{array}{r}4.354 \\
(25)\end{array}$ & $\begin{array}{r}6 \\
(1)\end{array}$ \\
\hline Valles Centrales & 3.400 & 0 & $\begin{array}{l}178 \\
(28)\end{array}$ & $\begin{array}{l}581 \\
(46)\end{array}$ & $\begin{array}{l}205 \\
(15)\end{array}$ & 0 \\
\hline $\begin{array}{l}\text { Montañas y Valles } \\
\text { del Centro }\end{array}$ & 8.399 & 0 & $\begin{array}{l}77 \\
(7)\end{array}$ & $\begin{array}{r}1.063 \\
(37)\end{array}$ & $\begin{array}{l}577 \\
(15)\end{array}$ & 0 \\
\hline $\begin{array}{l}\text { Depresión Ístmica } \\
\text { de Tehuantepec }\end{array}$ & 2.054 & 0 & $\begin{array}{r}8 \\
(1)\end{array}$ & $\begin{array}{r}221 \\
(5)\end{array}$ & $\begin{array}{r}130 \\
(2)\end{array}$ & $\begin{array}{r}3 \\
(1)\end{array}$ \\
\hline $\begin{array}{l}\text { Sierra Madre del } \\
\text { Sur de Oaxaca y } \\
\text { Chiapas }\end{array}$ & 6.211 & 0 & $\begin{array}{l}40 \\
(2)\end{array}$ & $\begin{array}{r}1.698 \\
(5)\end{array}$ & $\begin{array}{r}1.971 \\
(4)\end{array}$ & 0 \\
\hline $\begin{array}{l}\text { Sierra Madre del } \\
\text { Sur }\end{array}$ & 9.544 & 0 & $\begin{array}{l}1.148 \\
(102)\end{array}$ & $\begin{array}{r}4.786 \\
(63)\end{array}$ & $\begin{array}{l}641 \\
(22)\end{array}$ & $\begin{array}{l}14 \\
(1)\end{array}$ \\
\hline $\begin{array}{l}\text { Planicie Costera } \\
\text { del Pacífico }\end{array}$ & 11.182 & $\begin{array}{r}176 \\
(2)\end{array}$ & $\begin{array}{r}1.464 \\
(42)\end{array}$ & $\begin{array}{r}2.360 \\
(40)\end{array}$ & $\begin{array}{r}445 \\
(9)\end{array}$ & $\begin{array}{r}226 \\
(7)\end{array}$ \\
\hline $\begin{array}{l}\text { Planicie Costera } \\
\text { de Tehuantepec }\end{array}$ & 5.042 & 0 & 0 & $\begin{array}{r}327 \\
(5)\end{array}$ & 0 & $\begin{array}{l}60 \\
(1)\end{array}$ \\
\hline
\end{tabular}


Cuadro 3. Superficie de superposición y porcentaje respecto a la superficie total del estado, de las áreas protegidas en diferentes iniciativas de conservación.

Overlap surface and percentage in relation to the state's total surface, of the protected areas at different conservation initiatives.

\begin{tabular}{|c|c|c|c|c|c|c|c|c|c|}
\hline & $\begin{array}{c}\text { Áreas } \\
\text { protegidas }\end{array}$ & \multicolumn{2}{|c|}{$\begin{array}{l}\text { Áreas voluntarias } \\
\text { de conservación }\end{array}$} & \multicolumn{2}{|c|}{ Biodiversidad } & \multicolumn{2}{|c|}{ Hidrológicos } & \multicolumn{2}{|c|}{$\begin{array}{c}\text { Comunidades } \\
\text { con apoyo }\end{array}$} \\
\hline & & $\mathrm{km}^{2}$ & $\%$ & $\mathrm{~km}^{2}$ & $\%$ & $\mathrm{~km}^{2}$ & $\%$ & $\mathrm{~km}^{2}$ & $\%$ \\
\hline Áreas protegidas & - & 0 & 0 & $2.687,9$ & 4,92 & 572,9 & 1,05 & 133,6 & 0,24 \\
\hline Áreas voluntarias de conservación & & & & 6,4 & 0,01 & 289,9 & 0,53 & $1.924,0$ & 2,01 \\
\hline Biodiversidad & & & & & & 100,2 & 0,18 & $1.234,8$ & 2,26 \\
\hline Hidrológicos & & & & & & & & $4.170,4$ & 7,63 \\
\hline Comunidades con apoyo & & & & & & & & - & - \\
\hline
\end{tabular}

Cuadro 4. Porcentajes de los tipos de vegetación según la serie III del Inventario Nacional Forestal, representados en las iniciativas de conservación de Oaxaca, México.

Percentages of vegetation types according to the III series by the Inventario Nacional Forestal, represented in the conservation initiatives in Oaxaca, Mexico.

\begin{tabular}{|c|c|c|c|c|c|}
\hline \multirow[t]{2}{*}{ Tipo de vegetación } & $\begin{array}{c}\text { Áreas } \\
\text { protegidas }\end{array}$ & $\begin{array}{l}\text { Áreas voluntarias } \\
\text { de conservación }\end{array}$ & $\begin{array}{c}\text { Comunidades } \\
\text { apoyadas 2003-2007 }\end{array}$ & $\begin{array}{c}\text { Pagos } \\
\text { biodiversidad }\end{array}$ & $\begin{array}{c}\text { Pagos } \\
\text { hidrológicos }\end{array}$ \\
\hline & \multicolumn{5}{|c|}{$\%$} \\
\hline $\begin{array}{l}\text { Bosque bajo-abierto con vegetación } \\
\text { secundaria arbustiva y herbácea }\end{array}$ & 0,00 & 0,00 & 0,00 & 0,00 & 0,00 \\
\hline Bosque de encino & 19,80 & 0,00 & 3,56 & 9,80 & 5,32 \\
\hline $\begin{array}{l}\text { Bosque de encino con vegetación } \\
\text { secundaria arbustiva y herbácea }\end{array}$ & 5,02 & 0,00 & 2,20 & 2,37 & 3,44 \\
\hline Bosque de oyamel con ayarín y cedro & 0,00 & 0,00 & 0,06 & 0,04 & 0,02 \\
\hline Bosque de pino & 0,00 & 0,00 & 4,76 & 5,55 & 6,55 \\
\hline $\begin{array}{l}\text { Bosque de pino con vegetación } \\
\text { secundaria arbustiva y herbácea }\end{array}$ & 0,20 & 0,00 & 2,70 & 1,93 & 3,70 \\
\hline Bosque de pino-encino y encino-pino & 7,13 & 1,05 & 22,21 & 21,27 & 22,11 \\
\hline $\begin{array}{l}\text { Bosque de pino-encino y encino-pino } \\
\text { con vegetación secundaria arbustiva y } \\
\text { herbácea }\end{array}$ & 0,60 & 0,00 & 4,55 & 4,10 & 10.07 \\
\hline Bosque de táscate & 1,81 & 0,00 & 0,01 & 0,57 & 0,00 \\
\hline $\begin{array}{l}\text { Bosque de táscate con vegetación } \\
\text { secundaria arbustiva y herbácea }\end{array}$ & 0,32 & 0,00 & 0,00 & 0,05 & 0,00 \\
\hline Bosque mesófilo de montaña & 0,13 & 24,56 & 12,65 & 3,67 & 9,86 \\
\hline $\begin{array}{l}\text { Bosque mesófilo de montaña con } \\
\text { vegetación secundaria arbustiva y } \\
\text { herbácea }\end{array}$ & 0,03 & 3,77 & 6,52 & 6,10 & 6,45 \\
\hline Chaparral & 0,18 & 0,00 & 0,55 & 0,20 & 0,01 \\
\hline Manglar & 0,86 & 0,00 & 0,00 & 0,22 & 1,22 \\
\hline Matorral crasicaule & 3,70 & 0,00 & 0,00 & 0,59 & 0,00 \\
\hline $\begin{array}{l}\text { Matorral crasicuale con vegetación } \\
\text { secundaria }\end{array}$ & 0,48 & 0,00 & 0,00 & 0,08 & 0,00 \\
\hline Matorral desértico rosetófilo & 0,01 & 0,00 & 0,00 & 0,08 & 0,00 \\
\hline $\begin{array}{l}\text { Matorral desértico rosetófilo con } \\
\text { vegetación secundaria }\end{array}$ & 0,05 & 0,00 & 0,00 & 0,00 & 0,00 \\
\hline
\end{tabular}


Continuación Cuadro 4

\begin{tabular}{|c|c|c|c|c|c|}
\hline Matorral espinoso tamaulipeco & 0,12 & 0,00 & 0,00 & 0,03 & 0,00 \\
\hline Palmar & 1,14 & 0,16 & 0,02 & 0,95 & 0,02 \\
\hline Pastizal natural y pastizal-huizachal & 3,24 & 0,00 & 0,00 & 0,65 & 0,00 \\
\hline Popal-tular & 0,00 & 0,00 & 0,00 & 0,00 & 0,02 \\
\hline Pradera de alta montaña & 0,00 & 0,00 & 0,03 & 0,00 & 0,07 \\
\hline Sabana & 0,00 & 0,00 & 3,03 & 0,00 & 0,70 \\
\hline Selva alta y mediana perennifolia & 0,01 & 16,31 & 8,04 & 18,22 & 7,94 \\
\hline $\begin{array}{l}\text { Selva alta y mediana perennifolia con } \\
\text { vegetación secundaria arbustiva y } \\
\text { herbácea }\end{array}$ & 0,01 & 9,33 & 3,27 & 10,00 & 5,53 \\
\hline Selva alta y mediana subperennifolia & 0,79 & 0,00 & 5,47 & 0,19 & 3,54 \\
\hline $\begin{array}{l}\text { Selva alta y mediana subperennifolia } \\
\text { con vegetación secundaria arbustiva y } \\
\text { herbácea }\end{array}$ & 1,08 & 0,00 & 1,32 & 0,18 & 1,16 \\
\hline $\begin{array}{l}\text { Selva baja caducifolia y } \\
\text { subcaducifolia }\end{array}$ & 46,78 & 38,08 & 3,85 & 10,86 & 5,06 \\
\hline $\begin{array}{l}\text { Selva baja caducifolia y } \\
\text { subcaducifolia con vegetación } \\
\text { secundaria arbustiva y herbácea }\end{array}$ & 2,70 & 4,49 & 0,69 & 0,75 & 2,24 \\
\hline $\begin{array}{l}\text { Selva baja espinosa con vegetación } \\
\text { secundaria arbustiva y herbácea }\end{array}$ & 0,00 & 0,00 & 0,00 & 0,00 & 0,02 \\
\hline $\begin{array}{l}\text { Selva mediana caducifolia y } \\
\text { subcaducifolia }\end{array}$ & 2,91 & 1,20 & 7,77 & 1,20 & 2,13 \\
\hline $\begin{array}{l}\text { Selva mediana caducifolia y } \\
\text { subcaducifolia con vegetación } \\
\text { secundaria arbustiva y herbácea }\end{array}$ & 0,30 & 0,00 & 6,69 & 0,22 & 2,63 \\
\hline Vegetación con dunas costeras & 0,39 & 0,12 & 0,00 & 0,07 & 0,12 \\
\hline $\begin{array}{l}\text { Vegetación de galería con bosque, } \\
\text { selva y vegetación de galería }\end{array}$ & 0,20 & 0,00 & 0,03 & 0,05 & 0,00 \\
\hline Vegetación halófila y gipsófila & 0,00 & 0,93 & 0,00 & 0,00 & 0,05 \\
\hline
\end{tabular}

secundaria arbustiva y herbácea, popal-tular y matorral desértico rosetófilo con vegetación secundaria. Mientras que el bosque de pino-encino y la selva baja caducifolia y subcaducifolia se encontraron en más de $50 \%$ (cuadro 4). Por otro lado, 16 tipos de vegetación estuvieron representados en las cinco diferentes iniciativas de conservación y tres tipos de vegetación (selva baja espinosa con vegetación secundaria y arbustiva y herbácea, popal-tular y matorral desértico rosetófilo con vegetación secundaria) estuvieron presentes en solamente una de las iniciativas de conservación (dos en las comunidades apoyadas con pagos por servicios ambientales hidrológicos y la otra en un área natural protegida; cuadro 4).

Las comunidades apoyadas con pagos por servicios ambientales por biodiversidad fueron las que representaron un mayor número de tipos de vegetación (29), así como las áreas naturales protegidas (28); en cambio, las que mostraron menor variedad de tipos de vegetación fueron las áreas voluntarias de conservación (11), esto debido a que ocupan un área menor que las demás iniciativas (cuadro 4).
En la clasificación de los tipos de vegetación de acuerdo con su estado de conservación (vegetación primaria y vegetación secundaria) para cada tipo de iniciativa, se mostró que las áreas voluntarias de conservación albergan un mayor porcentaje de vegetación primaria dentro de sus superficies $(89,2 \%)$. Las comunidades con pagos por servicios ambientales hidrológicos son los que poseen el mayor porcentaje de vegetación secundaria (35, 2\%; figura 3 ).

Se analizaron los datos por medio de un análisis de Varianza de Medidas Repetidas y se encontraron diferencias significativas entre las coberturas de los tipos de vegetación en las diferentes iniciativas de conservación $\left(X^{2}=43,92\right.$, g.l. 4, $\left.P<0,001\right)$. Al aislar los diferentes grupos (iniciativas de conservación), no se encontraron diferencias significativas en dos de las comparaciones: por un lado, pagos por biodiversidad y pagos hidrológicos y, por otro lado entre áreas protegidas y comunidades apoyadas durante 2003-2007, mientras que en las restantes ocho comparaciones sí hubo diferencias significativas $(P<0,05)$. 


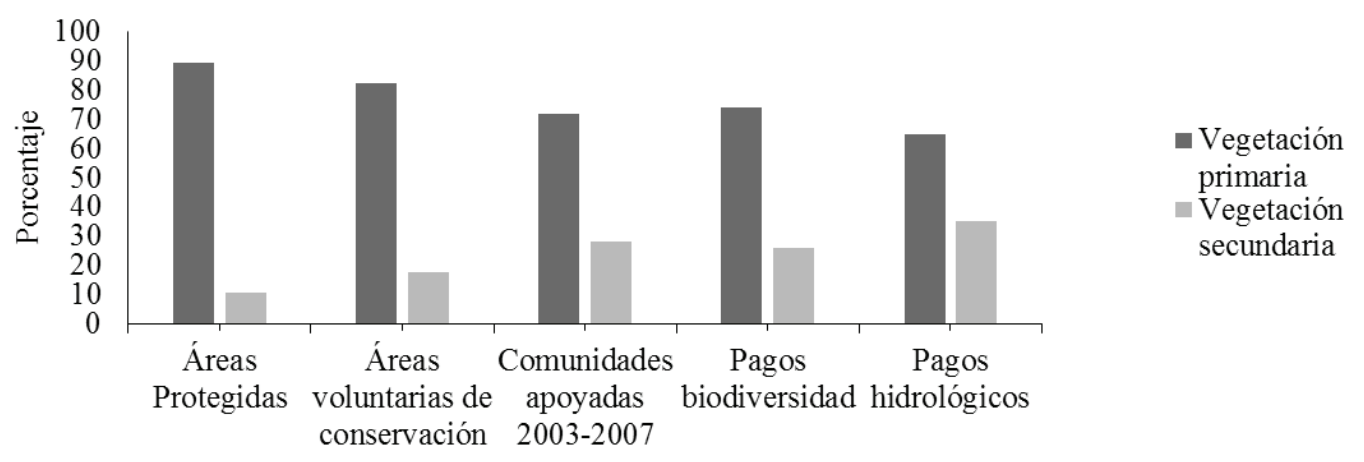

Iniciativas de conservación

Figura 3. Porcentaje de cobertura vegetal según su estado de conservación.

Percentage of vegetal coverage referred to their conservancy mode.

\section{DISCUSIÓN}

El conjunto de iniciativas de conservación muestra una cobertura de aproximada del $40 \%$ del estado (figura 2, cuadro 2). La representación de los tipos de vegetación presente en las iniciativas de conservación, en su conjunto, es notoriamente mayor, a la representatividad que se obtiene, al considerar solamente a las áreas protegidas decretadas. Esto demuestra, claramente, que es necesario incorporar iniciativas de conservación regionales o locales, para complementar adecuadamente la representatividad de los tipos de vegetación que cubren las áreas protegidas decretadas, conservando así una mayor biodiversidad (CONABIO et al. 2007). Para el caso de Oaxaca, la red de áreas protegidas decretadas no incluye todos los tipos de vegetación; por tanto, es necesario incorporar otras iniciativas de conservación, como las áreas voluntarias de conservación, que hacen una valiosa aportación de la representatividad de tipos de vegetación.

Las comunidades que reciben pagos por servicios ambientales por biodiversidad y por servicios hidrológicos, muestran una superposición importante con otras iniciativas de conservación, particularmente en la provincia fisiográfica de la Sierra Madre de Oaxaca, debido a que esta parte del estado es donde existen una mayor cantidad de áreas voluntarias de conservación (figura 2). Las comunidades que reciben pagos por servicios ambientales son las que más se superponen con otras, debido a que son los que cuentan con mayor superficie (figura 2).

Las áreas voluntarias de conservación protegen una superficie mayor en Oaxaca, en comparación con las áreas naturales protegidas estatales, lo que resalta la importancia de las organizaciones comunitarias indígenas en la conservación estatal, ya que, además, se trata de áreas conservadas producto de la iniciativa de las propias comunidades, con lo que se atenúan los conflictos sociales y políticos, por el control y el uso de los recursos. Además, se estimu- lan los procesos participativos, ya que a menudo no hacen distinción entre objetivos de conservación biológicos, económicos o sociales (Little 1994, Western y Wright 1994, Ghimire y Pimbert 1997, Wilshusen et al. 2002, Berkes 2009). Dichas áreas también resultan importantes ya que integran iniciativas de restauración ecológica donde hay vegetación secundaria (Alix-García et al. 2003).

Entre las áreas voluntarias de conservación, se han documentado algunos esfuerzos efectivos en cuanto a la conservación y restauración de ecosistemas. Un ejemplo es el caso de la Unión Zapoteca-Chinanteca (Sierra Madre de Oaxaca), en cuyas tierras se ha incrementado la cobertura forestal en 500 ha, después de 18 años de reforestación comunitaria y de la regulación de la agricultura en áreas boscosas (Bray et al. 2003). Otro ejemplo, es el de la comunidad de Santa Catarina Ixtepeji (Sierra Madre de Oaxaca), donde en la década de 1950 se encontraba la fábrica de papel Tuxtepec, que tuvo por tres décadas la concesión del Estado para explotar los bosques de la comunidad. En 1982, una vez terminado dicho plazo, la comunidad decidió revocar el permiso y formó una unidad propia de explotación forestal, con manejo sustentable, en donde establecieron un área dedicada a la conservación (Garnica et al. 2006). Estos casos exitosos sugieren que las áreas comunales de conservación generan tanto beneficios sociales como económicos (Bray et al. 2003).

Varios ordenamientos territoriales comunitarios se han desarrollado en comunidades que ya contaban con áreas protegidas previas, como en el caso de Santa María Tonameca, en el municipio de Pochutla, en la provincia de la Planicie Costera del Pacífico que, desde 2006, instauró una reserva natural comunitaria con apoyo del proyecto Conservación de la Biodiversidad por Comunidades Indígenas (COINBIO) de la Comisión Nacional Forestal. Esta reserva se encuentra dividida en siete secciones con una superficie total de $77,42 \mathrm{~km}^{2}$ e incluye la participación de las comunidades de Juan Diego, Cerrón, San Antonio, Po- 
trero-Mazunte, Corral de Piedra, Laguna Tonameca y Río Tonameca-San Francisco. Asimismo, algunas comunidades sin áreas protegidas han desarrollado otras acciones y avances importantes en conservación, como la comunidad Santa María Yucuhiti, en el municipio de Tlaxiaco, en la provincia de Montañas y Valles de Occidente del estado (figura 1) que instauró una campaña de reforestación, debido a que existe un alto índice de deforestación causado por plagas, lo que además causa erosión del suelo y en la comunidad hacen uso del bosque para plantaciones de café orgánico, el cual comercializan (López 2010).

La provincia fisiográfica de la Depresión del Balsas, que es la segunda con menor extensión, presenta sólo una iniciativa de conservación, de pagos por servicios ambientales hidrológicos. Esto contrasta con la provincia de la Fosa de Tehuacán que es la de menor extensión y presenta cuatro de las cinco iniciativas de conservación (cuadro 2). Lo anterior demuestra la forma en que se da la redundancia de los esfuerzos de conservación en Oaxaca (cuadro 3) en algunas regiones, mientras que en otras ya zonas importantes sin ningún tipo de protección. Es importante resaltar que en la provincia de la Sierra Madre de Oaxaca se encuentran todos los tipos de iniciativas de conservación; esto se debe, además de su extensión (la segunda más grande después de la de Montañas y Valles de Occidente) y a que es una de las más estudiadas y conocidas por su proximidad y acceso a través de la capital del estado (figura 1).

Aún existen extensas regiones del estado que no incluyen o incluyen muy pocas iniciativas de conservación, como la provincia de la Planicie Costera de Tehuantepec, en donde se localizan exclusivamente áreas certificadas privadas, dedicadas al cultivo de café de sombra. Esta región contiene una alta concentración de especies endémicas de flora y de fauna, además de que es el límite de distribución de muchos vertebrados, debido a las características fisiográficas particulares del lugar (García-Mendoza et al. 2004).

Las iniciativas de conservación muestran una representatividad diferencial de los distintos tipos de vegetación. Por ejemplo, $46,8 \%$ de la superficie de las áreas naturales protegidas y $38,1 \%$ de la de las áreas voluntarias de conservación protegen selva baja caducifolia y subcaducifolia. Es importante resaltar que en las áreas voluntarias de conservación se observa el porcentaje más alto de bosque mesófilo de montaña con $24,6 \%$ y conservan en su mayoría bosques de pino-encino y selva baja caducifolia y subcaducifolia (cuadro 4; Ortega-del Valle et al. 2010). Las comunidades apoyadas por la Comisión Nacional Forestal del 2003 al 2007 y las de pagos por servicios ambientales de biodiversidad e hidrológicos, conservan en su mayoría bosque de pino-encino; esto se debe a que es el tipo de vegetación con mayor cobertura estatal y el de mayor interés para el aprovechamiento forestal (cuadro 4; Ortega-del Valle et al. 2010).

Las diferentes iniciativas de conservación, en conjunto, conservan 35 de los 36 tipos de vegetación representados en Oaxaca (INEGI 2007), en tanto que, 35,5\% de los tipos de vegetación del estado se encuentran en las cinco diferentes iniciativas de conservación (cuadro 4). Esto sugiere que el conjunto de las iniciativas ofrecen una mejor conservación de los tipos de vegetación que de manera separada y, de manera indirecta, de la biodiversidad de Oaxaca. Además, el conjunto de las iniciativas de conservación poseen más de $50 \%$ de su superficie cubierta con vegetación primaria (figura 3). De los cuatro tipos de vegetación que cuentan con una extensión menor al 0,05\% en las diversas iniciativas de conservación, tres están mezcladas con vegetación secundaria por lo que esta subrepresentación no afecta de manera substancial; el otro tipo de vegetación es el tularpopal, que corresponde a un tipo de humedal asociado a aguas pantanosas o agua dulce estancada (INECC 2009). La iniciativa que protege más tipos de vegetación son las comunidades apoyadas con pagos por servicios ambientales por biodiversidad (cuadro 4), aunque son las que poseen un mayor porcentaje de cobertura transformada (figura 3). Por otro lado, aunque las áreas voluntarias de conservación sólo albergan 11 tipos de vegetación, contribuyen de manera importante a la conservación del bosque mesófilo de montaña, que es uno de los ecosistemas probablemente más amenazado del país. Este ecosistema, además de albergar una gran diversidad de flora y fauna en relación a la reducida área que ocupa, contiene una alta endemicidad y es un importante captador de recursos hidrológicos y de nutrientes (CONABIO 2010). Además este tipo de iniciativa de conservación es la que posee un mayor porcentaje de superficie con vegetación primaria (figura 3), lo que indica que estas áreas se encuentran en un buen estado de conservación. Es importante destacar que los diferentes tipos de vegetación son fuente crucial de recursos que son útiles económicamente, para la vida cotidiana de los pobladores que los habitan (maderables, medicinales, ceremoniales y alimentarios). Es por esto, que las áreas voluntarias de conservación son iniciativas de conservación que resaltan en importancia debido a que, en Oaxaca, la mayoría de los bosques son controlados y manejados por los residentes locales, lo que puede proponerse como un modelo para ser impulsado en otras regiones del país (Martin et al. 2011).

Este trabajo muestra que una visión integral de las diferentes iniciativas de conservación permite observar que éstas representan adecuadamente los tipos de vegetación primaria, aunque no hay evidencia de qué tanto conservan la biodiversidad de Oaxaca. En este estado se aprecian esfuerzos importantes en iniciativas de conservación, inclusive merecedoras de varios reconocimientos a nivel internacional y señaladas como modelo para otras áreas geográficas (Bray et al. 2003). En este contexto, es necesario reconocer la contribución de académicos, organizaciones no gubernamentales y los cuerpos técnicos de las comunidades indígenas, para incorporar su conocimiento a las actividades productivas de las comunidades y diversificarlas, a fin de lograr mejores perspectivas para la conservación de la biodiversidad y un manejo sustentable de los recursos naturales (Bocco et al. 2000, Berkes y Davidson-Hunt 2006). 


\section{AGRADECIMIENTOS}

La primera autora agradece a CONACYT la beca (200469) otorgada para sus estudios de posgrado. Este trabajo es parte de la tesis doctoral de la primera autora en el Doctorado en Ciencias Biomédicas de la Universidad Nacional Autónoma de México. MB-S agradece a la COFAA, EDI del IPN, el apoyo brindado. Se agradece a Salvador Anta (ex gerente regional de la Región 5) de la Comisión Nacional Forestal por la ayuda en la obtención de datos.

\section{REFERENCIAS}

Alix-García J, A de Janvry, E Sodoulet. 2003. A tale of two communities: explaining deforestation in Mexico. Department of Agricultural \& Resource Economics, University of California at Berkeley CUDARE Working Papers 964: 1-37.

Berkes F. 2009. Community conserved areas: policy issues in historic and contemporary context. Conservation Letters 2: 19-24.

Berkes F, IJ Davidson-Hunt. 2006. Biodiversity, traditional management systems and cultural landscapes: examples from de Boreal forest of Canada. International Social Science Journal 58(187): 35-47.

Bocco G, A Velázquez, A Torres A. 2000. Ciencia, comunidades indígenas y manejo de recursos naturales. Un caso de investigación participativa en México. Interciencia 25(2): 64-70.

Borrini-Feyerabend G, A Kothari, G Oviedo. 2004. Indigenous and local communities and protected areas. Towards equality and enhaced conservation. World Comission on Protected Areas, Best Practice Protected Area Guidelines Series $\mathrm{N}^{\mathrm{o}}$ 11. Cambridge, United Kingdom. IUCN-The World Conservation Union. $111 \mathrm{p}$.

Bray DB, L Merino, P Negreros-Castillo, G Segura-Warnholtz, JM Torres-Rojo, HFM Vester. 2003. Mexico's communitymanaged forests as a global model for sustainable landscapes. Conservation Biology 17(3): 672-677.

CONABIO (Comisión Nacional para el Conocimiento y Uso de la Biodiversidad, MX). 2008. Áreas Protegidas en México. Consultado 12 jul. 2011. Disponible en http://www.biodiversidad.gob.mx/region/areasprot/enmexico.html

CONABIO (Comisión Nacional para el Conocimiento y Uso de la Biodiversidad, MX). 2010. El bosque mesófilo de montaña en México: Amenazas y oportunidades para su conservación y manejo sostenible. Distrito Federal, México. Comisión Nacional para el Conocimiento y Uso de la Biodiversidad. $197 \mathrm{p}$.

CONABIO, CONANP, TNC, Pronatura - FCF, UANL (Comisión Nacional para el Conocimiento y Uso de la Biodiverisdad, MX - Comisión Nacional de Áreas Naturales Protegidas, MX - The Nature Conservancy, US - Pronatura - Facultad de Ciencias Forestales, MX - Universidad Autónoma de Nuevo Léon, MX). 2007. Análisis de vacíos y omisiones en conservación de la biodiversidad terrestres de México: espacios y especies. México DF, México. Comisión Nacional para el Conocimiento y Uso de la Biodiversidad, Comisión Nacional de Áreas Naturales Protegidas, The Nature Conservancy-Programa México, Pronatura, A. C., Facultad de Ciencias Forestales, Universidad Autónoma de Nuevo Léon. 127 p.
CONANP (Comisión Nacional de Áreas Naturales Protegidas, MX). 2007. Listado de áreas certificadas. Consultado 20 nov. 2007. Disponible en http://www.conanp.gob.mx/que hacemos/listado_areas.php

CONANP (Comisión Nacional de Áreas Naturales Protegidas, MX). 2008. Áreas Naturales Protegidas. Consultado 25 nov. 2008. Disponible en http://www.conanp.gob.mx

DOF (Diario Oficial de la Federación, MX). 1992. Ley Agraria. Secretaría de Gobernación. Estados Unidos Mexicanos. Consultado 26 jun. 2014. Disponible en http://dof.gob.mx/ ley-reg.php

Elizondo C, D López. 2009. Las áreas voluntarias de conservación en Quintana Roo. Corredor Biológico Mesoamericano México. Serie acciones. No 6. México DF, México. Comisión Nacional para el Conocimiento y Uso de la Biodiversidad. $126 \mathrm{p}$.

García-Mendoza AJ, MJ Ordoñez, M Briones-Salas eds. 2004. Biodiversidad de Oaxaca. Instituto de Biología Universidad Nacional Autónoma de México-Fondo Oaxaqueño para la Conservación de la Naturaleza. México DF, México. World Wildlife Fund, México. 605 p.

Garnica SZ, M Martínez, T Fuentes. 2006. Informe de evaluación para la certificación del manejo forestal de: Comunidad Santa Catarina Ixtepeji en Oaxaca. Oaxaca, México. Oficina Regional de Smartwood. 106 p.

Ghimire KB, MP Pimbert. 1997. Social change and conservation: an overview of issues and concepts. In Ghimire KB, MP Pimbert eds. Social change and conservation. London, UK. Earthscan. p. 1-45.

INECC (Instituto Nacional de Ecología y Cambio Climático, MX). 2009. Los ecosistemas de México. Consultado 20 abr. 2015. Disponible en http://www.inecc.gob.mx/coneco-ch/382-hc-ecosistemas-mexico

INEGI (Instituto Nacional de Estadística y Geografía, MX). 2007. Carta de uso de suelo y vegetación. Serie III. 1: 250000. México.

LGEEPA. 2012. Ley General del Equilibrio Ecológico y la Protección al Ambiente. Últimas reformas publicadas DOF 29-05-2012. Cámara de Diputados del H. Congreso de la Unión. Secretaría General. Secretaría de Servicios Parlamentarios. Dirección General de Servicios de Documentación, Información y Análisis. Estados Unidos Mexicanos. Distrito Federal, México. 112 p.

Little PD. 1994. The links between participation and improved conservation: a review of issues and experiences. In Western D, RM Wright, SC Strum eds. Natural connections perspectives in community-based conservation. Washington DC, USA. Island Press. p. 347-372.

López LMD. 2010. Plan Municipal de Desarrollo 2011-2013. Municipio de Santa María Yucuhiti, Oaxaca. Oaxaca, México. Municipio de Santa María Yucuhiti. 119 p.

Martin GJ, CI Camacho, CA del Campo, S Anta, F Chapela, MA González. 2011. Indigenous and community conserved areas in Oaxaca, Mexico. Management and Environmental Quality: An International Journal 22(2): 250-260.

Mittermeier RA, P Robles, C Goettsch-Mittermeier. 1997. Megadiversidad: los países biológicamente más ricos del mundo. México DF, México. Cemex. 501 p.

Moguel P, VM Toledo. 1999. Biodiversity conservation in traditional coffee systems of Mexico. Conservation Biology 13(1): 11-21. 
Ortega-del Valle D, G Sánchez, C Solano, MA Huerta, V Meza, C Galindo-Leal. 2010. Áreas de conservación certificadas en el estado de Oaxaca. Oaxaca, México. World Wildlife Fund-Comisión Nacional de Áreas Naturales Protegidas. $131 \mathrm{p}$.

Ortiz-Pérez MA, JR Hernández, JM Figueroa. 2004. Reconocimiento fisiográfico y geomorfológico. In García-Mendoza AJ, MA Ordoñez, M Briones-Salas eds. Biodiversidad de Oaxaca. México DF, México. Instituto de Biología, Universidad Nacional Autónoma de México-Fondo Oaxaqueño para la Conservación de la Naturaleza-World Wildlife Fund. p. 43-54.

Sarukhán J, J Larson. 2001. When the commons become less tragic: land tenure, social organization, and fair trade in
Mexico. In Burger J, E Ostrom, R Norgaard, D Policansky, BD Goldstein eds. Protecting the commons: A framework for resource management in the Americas. Washington DC, USA. Island Press. 360 p.

Western D, RM Wright. 1994. The background to community based conservation. In Western D, RM Wright, SC Strum eds. Natural connections perspectives in community-based conservation. Washington DC, USA. Island Press. p. 1-14.

Wilshusen PR, SR Brechin, CL Fortwangler, PC West. 2002. Reinventing a square wheel: critique a resurgent "protection paradigm” in International biodiversity conservation. Society and Natural resources 15: 17-40.

Zar JH. 1984. Bioestatistical Analysis. 2 ed. New York, USA. Prentice-Hall, Englegood Cliffs. 718 p.

Recibido: 09.01.14

Aceptado: 25.02.15 